\title{
SOME COVARIANTS RELATED TO STEINER SURFACES
}

\author{
FRANCK ARIES, EMMANUEL BRIAND AND CLAUDE BRUCHOU
}

\begin{abstract}
A Steiner surface is the generic case of a quadratically parameterizable quartic surface used in geometric modeling. This paper studies quadratic parameterizations of surfaces under the angle of Classical Invariant Theory. Precisely, it exhibits a collection of covariants associated to projective quadratic parameterizations of surfaces, under the actions of linear reparameterization and linear transformations of the target space. Each of these covariants comes with a simple geometric interpretation.

As an application, some of these covariants are used to produce explicit equations and inequalities defining the orbits of projective quadratic parameterizations of quartic surfaces.
\end{abstract}

\section{INTRODUCTION}

This paper deals with quadratically parameterizable quartic surfaces of $\mathbb{R}^{3}$, that is surfaces of degree 4 admitting a parameterization of the form:

$$
\begin{aligned}
& \mathbb{R}^{2} \quad \longrightarrow \quad \mathbb{R}^{3} \\
& \left(x_{1}, x_{2}\right) \longmapsto\left(\frac{F_{1}\left(x_{1}, x_{2}\right)}{F_{0}\left(x_{1}, x_{2}\right)}, \frac{F_{2}\left(x_{1}, x_{2}\right)}{F_{0}\left(x_{1}, x_{2}\right)}, \frac{F_{3}\left(x_{1}, x_{2}\right)}{F_{0}\left(x_{1}, x_{2}\right)}\right)
\end{aligned}
$$

where the $F_{i}$ are polynomial functions of degree at most 2. For generic $F_{i}$ 's, the parameterized surface obtained is called a Steiner surface, see section 2 for the precise definition.

Our general motivation for the study of Steiner surfaces is the following. Two of us (Franck Aries and Claude Bruchou) are interested in mathematical modeling of vegetation canopies (see [España et al., 1999] for more details). The detailed description of the architecture of vegetation canopies is critical for the modeling of many agricultural processes : the photosynthesis, the propagation of diseases from one organ to another or the radiative transfer. These processes involve a big amount of computations on geometric objects associated to each plant organ. Each geometric object can be approximated by a set of plane triangles, or more complex patches like bicubic. As underlined in several papers of geometric modeling ([Sederberg and Chen, 1995] [Coffman et al., 1996], [Aries et al., 2004]), Steiner patches are a possibly good compromise between triangles, which need to be very many for a good accuracy, and the eighteen degree surfaces associated to the bicubic parameterization, which raise problems of complexity. Unfortunately, one may meet singular, or close to singular parameterizations, that make computations unreliable. Thus one needs to know as much as possible about the geometry of the space of quadratic parameterizations.

The study of quadratic parameterizations is eased by considering, instead of the affine setting, the projective setting. This means considering the projective quadratic parameterizations of surfaces, that is the quadratic rational maps from the real projective plane $\mathbb{R} \mathbb{P}^{2}$

This paper will be published by Springer in the Proceedings of the Worshop COMPASS II. 
to the real projective space $\mathbb{R P}^{3}$. These maps are those of the form:

$$
\begin{aligned}
\Omega \subset \mathbb{R P}^{2} & \longrightarrow \mathbb{R P}^{3} \\
\left(x_{0}: x_{1}: x_{2}\right) & \longmapsto\left(f_{0}: f_{1}: f_{2}: f_{3}\right) .
\end{aligned}
$$

where the $f_{i}$ 's are quadratic forms in $x_{0}, x_{1}, x_{2}$ and $\Omega$ is a non-empty Zariski open subset of $\mathbb{R P}^{2}$.

The main topic of the present paper is the Invariant Theory of projective quadratic parameterizations under linear changes of coordinates of $\mathbb{R P} \mathbb{P}^{2}$ and $\mathbb{R} \mathbb{P}^{3}$. Precisely, we provide a collection of covariants with simple geometric interpretation.

Let us give a motivating problem: the discrimination between the different kinds of quadratic parameterizations of quartic surfaces. Let us make this precise. Consider a quadratic map as in (2). Its image in $\mathbb{R P}^{3}$ is not, in general, Zariski-closed. Consider its Zariski closure, it is an algebraic surface of degree at most 4 . Let $\mathcal{U}$ be the set of those maps for which it is a quartic, i.e. it has degree exactly 4 . Two elements of $\mathcal{U}$ are considered equivalent if one is obtained from the other by a linear reparameterization (linear change of coordinates in the domain $\mathbb{R P}^{2}$ ) and a projective transformation of the ambient space (linear change of coordinates in the codomain $\mathbb{R P}^{3}$ ). Then, as it is shown in [Coffman et al., 1996] and [Degen, 1996], there are finitely many equivalence classes in $\mathcal{U}$. The problem is to discriminate between these equivalence classes. Algorithmic solutions to this problem have been given in [Coffman et al., 1996] and [Aries et al., 2004]. Our paper proposes a new solution. It consists simply in providing polynomial equations and inequalities defining the equivalence classes ${ }^{1}$. The equivalence classes are actually orbits under the action of some group. Thus it is natural to look for the equations and inequalities among the objects provided by Classical Invariant Theory: the covariants. Then, the aforementioned problem of discrimination between orbits of parameterizations is solved as an application, by picking in our toolbox of covariants the most adapted ones.

The sequel of the paper is organized as follows: Section 2 recalls known facts about the classification of quadratic parameterizations of surfaces; Section 3 provides preliminaries on Classical Invariant Theory; Section 4 presents some geometrical features of Steiner surfaces, that will be helpful to present our collection of covariants; these covariants are introduced in Section 5; the last section, Section 6, presents the application of these covariants to the discrimination of classes of parameterizations.

\section{ORBITS OF QUADRATIC PARAMETERIZATIONS OF QUARTICS}

A quadratic rational map from $\mathbb{R P}^{2}$ to $\mathbb{R P}^{3}$ is determined by a homogeneous quadratic map $f$ from $\mathbb{R}^{3}$ to $\mathbb{R}^{4}$, that can be presented as a family of four real ternary quadratic forms:

$$
f=\left(f_{0}\left(x_{0}, x_{1}, x_{2}\right), f_{1}\left(x_{0}, x_{1}, x_{2}\right), f_{2}\left(x_{0}, x_{1}, x_{2}\right), f_{3}\left(x_{0}, x_{1}, x_{2}\right)\right) .
$$

Denote with $\mathcal{F}$ the space of all the quadruples of real ternary quadratic forms. Then, more precisely, quadratic rational maps from $\mathbb{R P}^{2}$ to $\mathbb{R P}^{3}$ can be identified with the elements of $\mathcal{F}$ considered modulo scalar multiplication, i.e. the projective space $\mathbb{P}(\mathcal{F})$. For $f \in \mathcal{F}$, we will denote with $[f]$ the corresponding element of $\mathbb{P}(\mathcal{F})$.

\footnotetext{
${ }^{1}$ Here is an example where the methods of [Coffman et al., 1996] and [Aries et al., 2004] are not directly applicable: suppose we are given a family of parameterizations, depending on a parameter $t$. Then, by mere specialization of the general equations and inequalities defining the classes, we are able to determine which values of $t$ give a parameterization in a given equivalence class.
} 
Now the group $G L(3, \mathbb{R})$ acts naturally on $\mathbb{R}^{3}$ (and $\mathbb{R}^{2}$ ), and thus on $\mathcal{F}$ (and $\mathbb{P}(\mathcal{F})$ ). The action on $\mathcal{F}$ is as follows: for $\theta \in G L(3, \mathbb{R})$,

$$
\theta(f)=f \circ \theta^{-1} \text {. }
$$

The induced action on $\mathbb{P}(\mathcal{F})$ corresponds to linear reparameterizations. There is also a natural action of the group $G L(4, \mathbb{R})$ on $\mathbb{R}^{4}$ (and $\mathbb{R} \mathbb{P}^{3}$ ), and thus on $\mathcal{F}$ (and $\mathbb{P}(\mathcal{F})$ ): for $\rho \in G L(4, \mathbb{R})$,

$$
\rho(f)=\rho \circ f
$$

We have thus an action of $G L(3, \mathbb{R}) \times G L(4, \mathbb{R})$ on $\mathcal{F}$ (and $\mathbb{P}(\mathcal{F})$ ). In the sequel, we will denote this group with $G$.

In $\mathbb{P}(\mathcal{F})$, the subset $\mathcal{U}$ of those projective parameterizations with the property that the Zariski closure of their image ${ }^{2}$ is a surface of degree 4 exactly, is invariant under $G$. It is also a Zariski dense open set. As said in the introduction, the decomposition of $\mathcal{U}$ into orbits is known ${ }^{3}$; see [Coffman et al., 1996], [Degen, 1996] and [Aries et al., 2004]. There are only six orbits. Table 1 provides the list of the orbits, with a representative for each.

\begin{tabular}{|c|c|}
\hline Orbit & Representative \\
\hline Ii & $\left(2 x_{1} x_{2}: 2 x_{0} x_{2}: 2 x_{0} x_{1}: x_{0}{ }^{2}+x_{1}{ }^{2}+x_{2}^{2}\right)$ \\
Iii & $\left(2 x_{1} x_{2}: 2 x_{0} x_{2}: 2 x_{0} x_{1}: x_{0}{ }^{2}-x_{1}^{2}+x_{2}^{2}\right)$ \\
Iiii & $\left(x_{0}{ }^{2}+x_{1}^{2}: x_{1}^{2}+x_{2}^{2}: x_{0} x_{2}: x_{1} x_{2}\right)$ \\
\hline IIi & $\left(x_{0}{ }^{2}-x_{1}^{2}: x_{0} x_{1}: x_{1} x_{2}: x_{2}^{2}\right)$ \\
IIii & $\left(x_{0} x_{2}-x_{1} x_{2}: x_{0}{ }^{2}: x_{1}^{2}: x_{2}^{2}\right)$ \\
\hline III & $\left(x_{0}{ }^{2}: x_{0} x_{2}-x_{1}^{2}: x_{1} x_{2}: x_{2}^{2}\right)$ \\
\hline
\end{tabular}

TABLE 1. Orbits of quadratic parameterizations of quartic surfaces.

Let us say a word about the connection between this problem and the analogous problem in the complex setting. Denote with $\mathcal{F}_{\mathbb{C}}$ the complexification of $\mathcal{F}$ : that is the space of families of four complex quadratic forms. Then $\mathbb{P}\left(\mathcal{F}_{\mathbb{C}}\right)$ represents the space of quadratic rational maps from the complex projective plane, $\mathbb{C P}^{2}$ to the complex projective threedimensional space, $\mathbb{C P}^{3}$. Let $\mathcal{U}_{\mathbb{C}}$ be the subset of those parameterizations whose image is a quartic surface. Then $\mathcal{U}$ is the trace of $\mathcal{U}_{\mathbb{C}}$ on $\mathbb{P}(\mathcal{F})$. This means that $\mathcal{U}=\mathcal{U}_{\mathbb{C}} \cap \mathbb{P}(\mathcal{F})$.

Let $G_{\mathbb{C}}=G L(3, \mathbb{C}) \times G L(4, \mathbb{C})$. This group acts naturally on $\mathcal{F}_{\mathbb{C}}$ and $\mathbb{P}\left(\mathcal{F}_{\mathbb{C}}\right)$, and also on $\mathcal{U}_{\mathbb{C}}$. The classification of the orbits of $\mathbb{P}(\mathcal{F})$ under $G$ is obtained by refining the classification of $\mathbb{P}\left(\mathcal{F}_{\mathbb{C}}\right)$ into orbits under $G_{\mathbb{C}}$ (see [Apery, 1987] for a modern reference about this classification in the complex setting). Precisely: if $\mathcal{O}$ is an orbit in $\mathbb{P}\left(\mathcal{F}_{\mathbb{C}}\right)$ under $G_{\mathbb{C}}$, then its trace (intersection with $\mathbb{P}(\mathcal{F})$ ) is a union of orbits under $G$. For instance, $\mathcal{U}_{\mathbb{C}}$ decomposes in three orbits: $\mathbb{I}_{\mathbb{C}}, \mathrm{II}_{\mathbb{C}}$ and $\mathrm{III}_{\mathbb{C}}$, and their respective traces on $\mathcal{U}$ are Ii $\cup$ Iii $\cup$ Iiii, $\mathrm{IIi} \cup \mathrm{IIii}$, and III.

It happens that there is one dense orbit in $\mathbb{P}\left(\mathcal{F}_{\mathbb{C}}\right)$ : that is Orbit $\mathbb{I}_{\mathbb{C}}$. Then a complex Steiner surface is just the image in $\mathbb{C P}^{3}$ of a parameterization in this orbit. It is always a Zariski closed quartic surface. By extension, the name "Steiner surface" is sometimes used

\footnotetext{
${ }^{2}$ We consider the set-theoretical image, and rule out the cases when the Zariski closure of the image is a double quadric (case 7 in Proposition 5 of [Aries et al., 2004]) or a plane counted four times.

${ }^{3}$ The determination of the orbits outside $\mathcal{U}$ is a different problem. See the references in [Coffman et al., 1996].

${ }^{4}$ One could, following some sources in the literature, refer to surfaces in Orbits $\mathrm{II}_{\mathbb{C}}$ and III $_{\mathbb{C}}$ as "degenerate" Steiner surfaces, but we will use the term Steiner surface only for the non-degenerate case, i.e. only for the elements of Orbit $\mathbf{I}_{\mathbb{C}}$.
} 
for the set of its real points ${ }^{5}$; that is a real quartic surface, Zariski closure of the image of a parameterization in Orbit Ii, Iii or Iiii.

\section{Preliminaries on Classical Invariant Theory}

The objects we will introduce in Section 5 are polynomial covariants for the action of $G$ on $\mathcal{F}$. We wish now to recall the general definition (we point out [Kraft and Procesi, 1996] and [Popov and Vinberg, 1994] as modern references for Classical Invariant Theory).

Let $\mathcal{G}$ be a group (we will apply what follows for $\mathcal{G}=G$ ), and let $W$ be some finitedimensional $\mathcal{G}$-module, that is: a vector space on which $\mathcal{G}$ acts linearly (we will have $W=\mathcal{F}$ ). Let $V$ be another finite-dimensional $\mathcal{G}$-module. A polynomial covariant ${ }^{6}$ of $W$ of type $V$ is a polynomial map $C$ from $W$ to $V$, equivariant with respect to $\mathcal{G}$. This means that:

$$
C(g(w))=g(C(w)) \quad \forall w \in W, \quad \forall g \in \mathcal{G} .
$$

This includes the (relative) invariants, which are the polynomial functions $I$ on $W$ such that for all $g \in \mathcal{G}$, there exists some scalar $c(g)$ such that:

$$
I(g(w))=c(g) \cdot I(w) \quad \forall w \in W .
$$

For $\mathcal{G}=G$ acting on $W=\mathcal{F}$, a polynomial covariant for the action of $G$ on $\mathcal{F}$ is a polynomial map from $\mathcal{F}$ to some $G$-module such that

$$
C\left(\rho \circ f \circ \theta^{-1}\right)=(\rho, \theta)(C(f))
$$

for all $\theta \in G L(3, \mathbb{R})$ and all $\rho \in G L(4, \mathbb{R})$.

Note that the zero set of any covariant is a $\mathcal{G}$-invariant set, that is a union of orbits.

We finish this section with some remarks. The covariants for $\mathcal{F}$ under $G$ are essentially the same as those of $\mathcal{F}_{\mathbb{C}}$ under $G_{\mathbb{C}}$ : the former are obtained by complexification of the latter ${ }^{7}$. From a classical theorem of Invariant Theory (see [Popov and Vinberg, 1994]), we know that the homogeneous covariants separate the orbits of $P\left(\mathcal{F}_{\mathbb{C}}\right)$ under $G_{\mathbb{C}}$ : this means that for any two orbits $\mathcal{O}_{1}$ and $\mathcal{O}_{2}$, there exists some homogeneous covariant vanishing on $\mathcal{O}_{1}$ and not on $\mathcal{O}_{2}$, or vice-versa. On the contrary, there is no guarantee in advance that we can separate the orbits of $\mathbb{P}(\mathcal{F})$ under $G$ using equations and inequalities involving only the covariants. We will be able to do it in Section 6 by using some derived objects.

\section{Some ElEMENTS OF GEOMETRY OF THE STEINER SURFACE}

To each of the covariants we will introduce is attached a simple geometric object associated to the quadratic parameterizations of the complex Steiner surface. This is, actually, what will guide us in the construction of the covariants.

We now introduce the main features of the Steiner surface (they can be found in [Salmon, 1915], parag. 554a). For $f \in \mathcal{F}$, denote with $S(f)$ the associated complex Steiner surface, that is the image of $\mathbb{C P}^{2}$ under $[f]$. Then:

- It is a quartic (its implicit equation has degree 4).

- Its singular locus is the union of three lines, that are double lines. They are concurrent: their intersection is the unique triple point of the Steiner surface.

\footnotetext{
${ }^{5}$ Nevertheless Steiner's Roman surface properly said corresponds to the Zariski closure of the image of a parameterization in Orbit Ii; see [Coffman et al., 1996].

${ }^{6}$ This is the modern meaning for covariant, which includes the classical notions of covariants, contravariants and mixed concomitants.

${ }^{7}$ For such issues of field of definition, see [Kraft and Procesi, 1996].
} 
- The intersection of $\mathcal{S}(f)$ with a tangent plane is a quartic curve that either decomposes as the union of two conics intersecting at four points, or as a double conic. The latter situation happens only for four tangent planes, that Salmon calls tropes. In the former situation, one of the four intersection points is the point of tangency; the three remaining points are the intersections of the plane with each of three double lines.

- Each trope is tangent to the Steiner surface along a conic, called a torsal conic ${ }^{8}$. There are thus four torsal conics.

- There is a unique quadric going through the four torsal conics. Let us call it the Associated Quadric.

- The dual (or "reciprocal") surface to $\mathcal{S}(f)$ (the surface of $\left(\mathbb{C P}^{3}\right)^{*}$ that is the Zariski closure of the set of all tangent planes to $\mathcal{S}(f)$ ) is a cubic surface, known as the Cayley Cubic Surface (see [Salmon, 1915]).

Also of interest are some facts connected to the quadratic parameterization $[f]$ (rather than to the Steiner surface $\mathcal{S}(f)$ itself):

- It is defined on the whole $\mathbb{C P}^{2}$.

- The direct image of each line of $\mathbb{C P}^{2}$ is a conic on $\mathcal{S}(f)$.

- The preimage of each conic drawn on $\mathcal{S}(f)$ is a straight line of $\mathbb{C P}^{2}$. As a consequence, the preimage of any tangent plane is a pair of lines. The lines are distinct, unless the plane is a trope.

- The four lines obtained as preimages of the four tropes (equivalently: of the torsal conics; yet equivalently: of the Associated Quadric) form a non-degenerate quadrilateral.

- The preimage of each of the singular lines of $\mathcal{S}(f)$ is a straight line of $\mathbb{C P}^{2}$. The 3 lines obtained this way are non concurrent: they form a (non-degenerate) triangle, that we call the Exceptional Triangle.

- The preimage of the triple point is the union of the vertices of the Exceptional Triangle.

- The parameterization is faithful (i.e. generically injective). Precisely, it is injective on the complement of the Exceptional Triangle in $\mathbb{C P}^{2}$.

\section{A COLLECTION OF COVARIANTS}

5.1. Preliminaries. This section presents the new contribution of the paper: a collection of homogeneous covariants for the action of $G$ on $\mathcal{F}$, with a simple geometric interpretation for each of them.

Let us start with some notations. Denote the canonical basis of $\mathbb{C}^{3}$ with $\lambda_{0}, \lambda_{1}, \lambda_{2}$ and its dual basis with $x_{0}, x_{1}, x_{2}$. Denote also the canonical basis of $\mathbb{C}^{4}$ with $\alpha_{0}, \alpha_{1}, \alpha_{2}, \alpha_{3}$ and its dual basis with $y_{0}, y_{1}, y_{2}, y_{3}$. Given two complex vector spaces $W$ and $V$, denote with $\operatorname{Pol}_{n}(W, V)$ the space of homogeneous polynomial maps from $W$ to $V$ of degree $n$. Denote also $\mathrm{Pol}_{n}(W)$ the space of polynomial homogeneous functions of degree $n$ over $W$. Otherwise stated,

$$
\mathrm{Pol}_{n}(W)=\mathrm{Pol}_{n}(W, \mathbb{C}) .
$$

For $f=\left(f_{0}, f_{1}, f_{2}, f_{3}\right) \in \mathcal{F}$, denote the coefficients of $f_{i}$ with $a_{i j}$ and $b_{i j}$, as follows:

$$
f_{i}=a_{i 0} x_{0}^{2}+a_{i 1} x_{1}^{2}+a_{i 2} x_{2}^{2}+2 b_{i 0} x_{1} x_{2}+2 b_{i 1} x_{0} x_{2}+2 b_{i 2} x_{0} x_{1} \text {. }
$$

\footnotetext{
${ }^{8}$ This is called a parabolic conic in [Coffman et al., 1996].
} 
Each of the homogeneous covariants we will present, considered up to a scalar, represents some geometric object associated to the parameterization $[f]$, according to its type (its space of values ${ }^{9}$ ). Note that the definition of this geometric object will be valid only in the case when $[f]$ parameterizes a Steiner surface.

We will meet covariants of the following types:

- Type $\operatorname{Pol}_{n}\left(\mathbb{C}^{4}\right)$ : such a covariant $C$ associates to $[f]$ a surface in $\mathbb{C P}^{3}$ (the zero locus of $C(f))$.

- Type $\operatorname{Pol}_{n}\left(\mathbb{C}^{3}\right)$ : such a covariant associates to $[f]$ a curve in $\mathbb{C P}^{2}$.

- Type $\operatorname{Pol}_{n}\left(\left(\mathbb{C}^{4}\right)^{*}\right)$ : such a covariant associates to $[f]$ a surface in $\left(\mathbb{C P}^{3}\right)^{*}$. If this surface is decomposable, that is a union of hyperplanes of $\left(\mathbb{C P}^{3}\right)^{*}$, then it also represents a finite collection of points in $\mathbb{C P}^{3}$ (the points corresponding to the hyperplanes by duality).

- Type $\operatorname{Pol}_{n}\left(\left(\mathbb{C}^{3}\right)^{*}\right)$ : such a covariant associates to $[f]$ a curve in $\left(\mathbb{C P}^{2}\right)^{*}$. If this curve is decomposable, then it also represents a finite collection of points in $\mathbb{C P}^{2}$.

- Type some space of functions $\operatorname{Pol}_{n}(W, V)$ between spaces $W, V$ among $\mathbb{C}^{3}, \mathbb{C}^{4}$ and their duals. Then the covariant associates to $[f]$ some family of curves or surfaces in $\mathbb{P}(V)^{*}$ parameterized by $\mathbb{P}(W)$.

- Type $\mathbb{C}$ : such a homogeneous covariant is just an invariant for the group $S L(3, \mathbb{C}) \times$ $S L(4, \mathbb{C})$. We will see that there is essentially only one invariant.

The geometric objects attached to some of the covariants we will present will be clear from their construction; for the rest, they can be found merely by evaluating the covariant on the representative of Orbit $\mathrm{I}_{\mathbb{C}}$ :

$$
\left(2 x_{1} x_{2}: 2 x_{0} x_{2}: 2 x_{0} x_{1}: x_{0}^{2}+x_{1}^{2}+x_{2}^{2}\right) .
$$

Table 2 recapitulates the list of covariants that will be now presented individually. The reader will find Maple procedures implementing the formulas that follow on the web page: http: //emmanuel.jean.briand.free.fr/publications/steiner/

5.2. Derivation of the covariants. Here we suppose that $[f]$ is in $\mathbf{I}_{\mathbb{C}}$, that is its image $\mathcal{S}(f)$ in $\mathbb{C P}^{3}$ is a complex Steiner surface.

For each covariant we indicate its type, and its degree with respect to the coefficients of the $f_{i}$ 's.

Tangent plane at the image of a point. Given a generic point $[x]$ in the parameter space $\mathbb{C P}^{2}$, we can consider the tangent plane to the Steiner surface $\mathcal{S}(f)$ at its image by $[f]$. It has equation $\Phi_{1}(f)(x)=0$, where

$$
\Phi_{1}=\frac{1}{8}\left|\begin{array}{cccc}
\partial_{0} f_{0} & \partial_{1} f_{0} & \partial_{2} f_{0} & y_{0} \\
\partial_{0} f_{1} & \partial_{1} f_{1} & \partial_{2} f_{1} & y_{1} \\
\partial_{0} f_{2} & \partial_{1} f_{2} & \partial_{2} f_{2} & y_{2} \\
\partial_{0} f_{3} & \partial_{1} f_{3} & \partial_{2} f_{3} & y_{3}
\end{array}\right| .
$$

Here $\partial_{i}$ stands for $\frac{\partial}{d x_{i}}$.

This covariant $\Phi_{1}$ has degree 3 and type $\operatorname{Pol}_{3}\left(\mathbb{C}^{3},\left(\mathbb{C}^{4}\right)^{*}\right)$. The geometric object associated to $\Phi_{1}(f)$ is a parameterization of the dual surface to $\mathcal{S}(f)$.

\footnotetext{
${ }^{9}$ Strictly speaking, the type should mention also the action of $G$ on this space. In all the cases we will meet, this action is a canonical action of $G$ on the space, or its product by some powers of the determinants of $\theta \in G L(3, \mathbb{R})$ and $\rho \in G L(4, \mathbb{R})$. These powers are easily determined from the degree of the covariant.
} 


\begin{tabular}{|c|c|c|c|}
\hline Symbol & Name & Degree & Type \\
\hline \multicolumn{3}{|c|}{ Invariants } & $\mathbb{C}$ \\
\hline$\Delta$ & Discriminant & 24 & $\mathbb{C}$ \\
\hline \multicolumn{3}{|c|}{ Families of objects } & \\
\hline$\overline{\Phi_{1}}$ & Tangent plane at the image of a point & 3 & $\mathrm{Pol}_{3}\left(\mathbb{C}^{3},\left(\mathbb{C}^{4}\right)^{*}\right)$ \\
\hline$\Phi_{2}$ & Linear plane spanned by the image of a line & 3 & $\mathrm{Pol}_{3}\left(\left(\mathbb{C}^{3}\right)^{*},\left(\mathbb{C}^{4}\right)^{*}\right)$ \\
\hline$\Phi_{3}$ & Correspondence line-line & 4 & $\mathrm{Pol}_{2}\left(\left(\mathbb{C}^{3}\right)^{*},\left(\mathbb{C}^{3}\right)^{*}\right)$ \\
\hline$\Phi_{6}$ & Preimage of a point on $\mathcal{S}(f)$ & 10 & $\mathrm{Pol}_{2}\left(\mathbb{C}^{4}, \mathbb{C}^{3}\right)$ \\
\hline \multicolumn{3}{|c|}{ Associated surfaces in $\mathbb{C P}^{3}$} & $\mathrm{Pol}_{n}\left(\mathbb{C}^{4}\right)$ \\
\hline$\overline{\Phi_{4}}$ & Implicit Equation & 12 & $n=4$ \\
\hline$\Phi_{5}$ & Associated Quadric & 6 & $n=2$ \\
\hline$\Phi_{9}$ & Union of the Tropes & 12 & $n=4$ \\
\hline$\Phi_{10}$ & Trihedron defined by the Double Lines & 21 & $n=3$ \\
\hline$\Phi_{12}$ & $\begin{array}{l}\text { Polar Plane } \Pi \text { of the Associated Quadric and the Triple } \\
\text { Point }\end{array}$ & 15 & $n=1$ \\
\hline \multicolumn{3}{|c|}{ Associated surfaces in $\left(\mathbb{C P}^{3}\right)^{*}$} & $\mathrm{Pol}_{n}\left(\left(\mathbb{C}^{4}\right)^{*}\right)$ \\
\hline$\Phi_{7}$ & Dual surface & 3 & $n=3$ \\
\hline$\Phi_{8}$ & Triple Point & 9 & $n=1$ \\
\hline \multicolumn{3}{|c|}{ Associated curves in $\mathbb{C P}^{2}$} & $\mathrm{Pol}_{n}\left(\mathbb{C}^{3}\right)$ \\
\hline$\Phi_{11}$ & Exceptional Triangle & 12 & $n=3$ \\
\hline$\Phi_{13}$ & Conic preimage of $\Pi$ & 16 & $n=2$ \\
\hline$\Phi_{15}$ & Quadrilateral preimage of the torsal conics & 8 & $n=4$ \\
\hline \multicolumn{3}{|c|}{ Associated surfaces of $\left(\mathbb{C P}^{2}\right)^{*}$} & $\mathrm{Pol}_{n}\left(\left(\mathbb{C}^{3}\right)^{*}\right)$ \\
\hline$\Phi_{14}$ & Dual conic to the preimage of $\Pi$ & 8 & $n=2$ \\
\hline
\end{tabular}

TABLE 2. List of the covariants presented in the paper.

Plane spanned by the image of a line. Consider a generic line $L$ in $\mathbb{C P}^{2}$, given by an equation

$$
\lambda(x)=\lambda_{0} x_{0}+\lambda_{1} x_{1}+\lambda_{2} x_{2}=0 .
$$

Its image under $f$ is a conic in $\mathbb{C P}^{3}$, spanning a plane, that is an element of $\left(\mathbb{C P}^{3}\right)^{*}$. This plane is always a tangent plane to $\mathcal{S}(f)$. It admits $\Phi_{2}(f)(\lambda)=0$ as an equation, with

$$
\Phi_{2}=\frac{1}{2}\left|\begin{array}{ccccccc}
a_{00} & a_{01} & a_{02} & 2 b_{00} & 2 b_{01} & 2 b_{02} & y_{0} \\
a_{10} & a_{11} & a_{12} & 2 b_{10} & 2 b_{11} & 2 b_{12} & y_{1} \\
a_{20} & a_{21} & a_{22} & 2 b_{20} & 2 b_{21} & 2 b_{22} & y_{2} \\
a_{30} & a_{31} & a_{32} & 2 b_{30} & 2 b_{31} & 2 b_{32} & y_{3} \\
\lambda_{0} & 0 & 0 & 0 & \lambda_{2} & \lambda_{1} & 0 \\
0 & \lambda_{1} & 0 & \lambda_{2} & 0 & \lambda_{0} & 0 \\
0 & 0 & \lambda_{2} & \lambda_{1} & \lambda_{0} & 0 & 0
\end{array}\right| .
$$

Note that the lines of the matrix in the determinant correspond to the equations:

$$
\begin{gathered}
f_{i}(x)=y_{i}, \quad i=0,1,2,3, \\
x_{j} \lambda(x)=0, \quad j=0,1,2,
\end{gathered}
$$

seen as linear in $x_{0}^{2}, x_{0} x_{1}, \ldots$

This function $\Phi_{2}$ is a covariant of degree 3 of type $\operatorname{Pol}_{3}\left(\left(\mathbb{C}^{3}\right)^{*},\left(\mathbb{C}^{4}\right)^{*}\right)$. The geometric object associated to $\Phi_{2}(f)$ is a (non-proper) parameterization of the the dual surface to $\mathcal{S}(f)$. 
Line whose image spans the same plane. As already mentioned, any section of $\mathcal{S}(f)$ by some of its tangent planes is a union of two conics. The preimage of each is a straight line in $\mathbb{C P}^{2}$.

Thus we have the following construction: take a generic line $L$ drawn in $\mathbb{C P}^{2}$, consider its image in $\mathbb{C P}^{3}$, this is a conic spanning a tangent plane. The preimage of this plane is made of the original line $L$, plus another one, $L^{\prime}$. The map $L \mapsto L^{\prime}$ is given by a covariant $\Phi_{3}$ of type $\operatorname{Pol}_{2}\left(\left(\mathbb{C}^{3}\right)^{*},\left(\mathbb{C}^{3}\right)^{*}\right)$. This covariant is defined by the formula

$$
\Phi_{3}=\left|\begin{array}{ccccccc}
a_{00} & a_{01} & a_{02} & 2 b_{00} & 2 b_{01} & 2 b_{02} & 0 \\
a_{10} & a_{11} & a_{12} & 2 b_{10} & 2 b_{11} & 2 b_{12} & 0 \\
a_{20} & a_{21} & a_{22} & 2 b_{20} & 2 b_{21} & 2 b_{22} & 0 \\
a_{30} & a_{31} & a_{32} & 2 b_{30} & 2 b_{31} & 2 b_{32} & 0 \\
\lambda_{0} & 0 & 0 & 0 & \lambda_{2} & \lambda_{1} & x_{0} \\
0 & \lambda_{1} & 0 & \lambda_{2} & 0 & \lambda_{0} & x_{1} \\
0 & 0 & \lambda_{2} & \lambda_{1} & \lambda_{0} & 0 & x_{2}
\end{array}\right| .
$$

It has degree 4 .

Implicit equation. The implicit equation of $\mathcal{S}(f)$ can be obtained as follows. Consider $\Phi_{1}(f)$ as a cubic polynomial in $x$ :

$$
\begin{gathered}
\Phi_{1}=\ell_{300}(y) x_{0}^{3}+\ell_{030}(y) x_{1}^{3}+\ell_{003}(y) x_{2}^{3}+3 \ell_{210}(y) x_{0}^{2} x_{1}+3 \ell_{201}(y) x_{0}^{2} x_{2} \\
+3 \ell_{120}(y) x_{1}^{2} x_{0}+3 \ell_{021}(y) x_{1}^{2} x_{2}+3 \ell_{102}(y) x_{2}^{2} x_{0}+3 \ell_{012}(y) x_{2}^{2} x_{1} \\
+6 \ell_{111}(y) x_{0} x_{1} x_{2} .
\end{gathered}
$$

Here the coefficients $\ell_{i j k}$ are linear forms in $y$, depending polynomially on $f$. Set

$$
\Phi_{4}=6^{3}\left|\begin{array}{lllllll}
a_{00} & a_{01} & a_{02} & b_{00} & b_{01} & b_{02} & y_{0} \\
a_{10} & a_{11} & a_{12} & b_{10} & b_{11} & b_{12} & y_{1} \\
a_{20} & a_{21} & a_{22} & b_{20} & b_{21} & b_{22} & y_{2} \\
a_{30} & a_{31} & a_{32} & b_{30} & b_{31} & b_{32} & y_{3} \\
\ell_{300} & \ell_{120} & \ell_{102} & \ell_{111} & \ell_{201} & \ell_{210} & 0 \\
\ell_{210} & \ell_{030} & \ell_{012} & \ell_{021} & \ell_{111} & \ell_{120} & 0 \\
\ell_{201} & \ell_{021} & \ell_{003} & \ell_{012} & \ell_{102} & \ell_{111} & 0
\end{array}\right| .
$$

Then $\Phi_{4}(f)$ is an implicit equation of $\mathcal{S}(f)$. And $\Phi_{4}$ is also a covariant. it has degree 12 and type $\mathrm{Pol}_{4}\left(\mathbb{C}^{4}\right)$. The attached geometric object is its zero locus, that is merely the surface itself.

This covariant has another property: it vanishes if and only if the parameterization admits a base point (this means that the $f_{i}$ 's have a common zero in $\mathbb{C P}^{2}$; thus it is revealed to be a resultant).

Formula (18) has been proposed in [Aries and Senoussi, 1997]. See [Brill, 1872], [Jouanolou, 1996], [Aries and Senoussi, 2001] for formulas close to this one, and proofs.

Associated Quadric. One produces a new covariant by the following contraction (see [Kraft and Procesi, 1996]) of $\Phi_{1}$ and $\Phi_{2}$ :

$$
\Phi_{5}=\frac{1}{6} \sum_{i, j, k} \frac{\partial^{3} \Phi_{1}}{d x_{i} d x_{j} d x_{k}} \frac{\partial^{3} \Phi_{2}}{d \lambda_{i} d \lambda_{j} d \lambda_{k}} .
$$

It has degree 6 and type $\mathrm{Pol}_{2}\left(\mathbb{C}^{4}\right)$. One finds (by evaluation on the representative of the dense orbit) that $\Phi_{5}(f)=0$ is an equation for the Associated Quadric. 
Preimage of a point of the Steiner surface. The map $[f]$ from $\mathbb{C P}^{2}$ to $\mathbb{C P}^{3}$ induced by $f$ is birational onto its image $\mathcal{S}(f)$ : its inverse is induced by the rational map $\left[\Phi_{6}(f)\right]: \mathbb{C P}^{3} \rightarrow$ $\mathbb{C P}^{2}$ where

$$
\Phi_{6}=6^{3}\left|\begin{array}{ccccccc}
a_{00} & a_{01} & a_{02} & b_{00} & b_{01} & b_{02} & 0 \\
a_{10} & a_{11} & a_{12} & b_{10} & b_{11} & b_{12} & 0 \\
a_{20} & a_{21} & a_{22} & b_{20} & b_{21} & b_{22} & 0 \\
a_{30} & a_{31} & a_{32} & b_{30} & b_{31} & b_{32} & 0 \\
\ell_{300} & \ell_{120} & \ell_{102} & \ell_{111} & \ell_{201} & \ell_{210} & \lambda_{0} \\
\ell_{210} & \ell_{030} & \ell_{012} & \ell_{021} & \ell_{111} & \ell_{120} & \lambda_{1} \\
\ell_{201} & \ell_{021} & \ell_{003} & \ell_{012} & \ell_{102} & \ell_{111} & \lambda_{2}
\end{array}\right| .
$$

This is a covariant of degree 10 and type $\mathrm{Pol}_{2}\left(\mathbb{C}^{4}, \mathbb{C}^{3}\right)$.

The dual surface. Consider the quadratic form $\alpha_{0} f_{0}+\cdots+\alpha_{3} f_{3}$ and take its discriminant (that is the determinant of its matrix):

$$
\Phi_{7}=\operatorname{Disc}\left(\alpha_{0} f_{0}+\alpha_{1} f_{1}+\alpha_{2} f_{2}+\alpha_{3} f_{3}\right) .
$$

The object obtained this way, $\Phi_{7}$, is a covariant. It has degree 3 and type $\operatorname{Pol}_{3}\left(\left(\mathbb{C}^{4}\right)^{*}\right)$. The zero locus of $\Phi_{7}(f)$ is the dual surface to $\mathcal{S}(f)$.

Triple point. A covariant of degree 9 and type $\mathbb{C}^{4} \cong \operatorname{Pol}_{1}\left(\left(\mathbb{C}^{4}\right)^{*}\right)$ is produced by contraction of $\Phi_{7}$ and $\Phi_{5}$ :

$$
\Phi_{8}=\sum_{i, j} \frac{\partial^{2} \Phi_{5}}{d y_{i} d y_{j}} \frac{\partial^{2} \Phi_{7}}{d \alpha_{i} d \alpha_{j}} .
$$

Write

$$
\Phi_{8}(f)=\tau_{0} \alpha_{0}+\tau_{1} \alpha_{1}+\tau_{2} \alpha_{2}+\tau_{3} \alpha_{3} .
$$

Then the associated geometric object is a point $\left(\tau_{0}: \tau_{1}: \tau_{2}: \tau_{3}\right)$ of $\mathbb{C P}^{3}$. One checks that this is exactly the triple point of $\mathcal{S}(f)$.

Discriminant. By evaluating $\Phi_{5}(f)$, the equation of the Associated Quadric, at $\Phi_{8}(f)$, the Triple Point, one gets a scalar:

$$
\Delta(f)=\Phi_{5}(f)\left(\Phi_{8}(f)\right) .
$$

This object $\Delta$ is a homogeneous covariant of degree 24 and type $\mathbb{C}$. Otherwise stated, this is a homogeneous invariant for $S L(3, \mathbb{C}) \times S L(4, \mathbb{C})$. One checks by direct computation that it is irreducible. From this and the existence of a dense orbit, it is not difficult to deduce that $\Delta$ is essentially the only invariant. This means that $\Delta$ generates the algebra of the invariants under $S L(3, \mathbb{C}) \times S L(4, \mathbb{C})$.

Union of the tropes. Set

$$
\Phi_{9}=\Phi_{4}+\Phi_{5}^{2} .
$$

This is a covariant of degree 12 and type $\mathrm{Pol}_{4}\left(\mathbb{C}^{4}\right)$, and thus $\Phi_{9}(f)$ represents some quartic surface in $\mathbb{C P}^{3}$. One checks that this surface is the union of the four tropes. 
Trihedron of the double lines. Remember the classical notion of polar: given an hypersurface of degree $d>1$ given by an equation $F\left(z_{0}, \ldots, z_{r}\right)=0$ and a point $\left(Z_{0}: \cdots: Z_{r}\right)$, the polar of the hypersurface and the point is the hypersurface of degree $d-1$ defined by the equation

$$
\sum_{i} Z_{i} \frac{\partial F}{d z_{i}}=0 .
$$

Then the polar of $\mathcal{S}(f)$ and the triple point $\tau(f)$ has equation $\varphi_{5}(f)=0$, where

$$
\Phi_{10}=\sum_{i=0}^{3} \tau_{i}(f) \frac{\partial \Phi_{4}}{d y_{i}} .
$$

(The $\tau_{i}$ 's are defined in Equation (23).) This way we get a covariant of degree 21 with type $\mathrm{Pol}_{3}\left(\mathbb{C}^{4}\right)$. One checks that its zero locus in $\mathbb{C P}^{3}$ is a union of three planes: they are the faces of the trihedron drawn by the singular lines of $\mathcal{S}(f)$.

Exceptional Triangle. Consider the discriminant of $\Phi_{3}$, quadratic form on $\left(\mathbb{C}^{3}\right)^{*}$ :

$$
\Phi_{11}=\frac{1}{8}\left|\begin{array}{ccc}
\frac{\partial^{2} \Phi_{3}}{d \lambda_{0}^{2}} & \frac{\partial^{2} \Phi_{3}}{d \lambda_{0} \lambda_{1}} & \frac{\partial^{2} \Phi_{3}}{d \lambda_{0} \lambda_{2}} \\
\frac{\partial^{2} \Phi_{3}}{d \lambda_{0} \lambda_{1}} & \frac{\partial^{2} \Phi_{3}}{d \lambda_{1}^{2}} & \frac{\partial^{2} \Phi_{3}}{d \lambda_{1} \lambda_{2}} \\
\frac{\partial^{2} \Phi_{3}}{d \lambda_{0} \lambda_{2}} & \frac{\partial^{2} \Phi_{3}}{d \lambda_{1} \lambda_{2}} & \frac{\partial^{2} \Phi_{3}}{d \lambda_{2}^{2}}
\end{array}\right| .
$$

This is a covariant of degree 12 and type $\mathrm{Pol}_{3}\left(\mathbb{C}^{3}\right)$. The zero locus of $\Phi_{11}(f)$ in $\mathbb{C P}^{2}$ is the Exceptional Triangle ${ }^{10}$.

Polar plane $\Pi$ of the Associated Quadric and the Triple Point. The polar surface of the Associated Quadric and the Triple Point is a plane, call it $\Pi$. It has equation $\Phi_{12}(f)=0$, where

$$
\Phi_{12}=\sum_{i=0}^{3} \tau_{i} \frac{\partial \Phi_{5}}{d y_{i}} .
$$

This is a covariant of degree 15 and type $\left(\mathbb{C}^{4}\right)^{*}=\mathrm{Pol}_{1}\left(\mathbb{C}^{4}\right)$.

Conic, preimage of $\Pi$. By merely substituting $y_{i}$ with $f_{i}(x)$ in $\Phi_{12}$, one finds a new covariant $\Phi_{13}$ :

$$
\Phi_{13}(f)(x)=\Phi_{12}(f)(f(x)) .
$$

The covariant $\Phi_{13}$ has degree 16 and type $\operatorname{Pol}_{2}\left(\mathbb{C}^{3}\right)$. Naturally, $\Phi_{13}(f)=0$ is the equation of the conic that is the preimage by $[f]$ of the section of $\mathcal{S}(f)$ by $\Pi(f)$.

Dual conic to the preimage of $\Pi$. In [Salmon, 1884], parag. 377 is shown a covariant $\Psi\left(q_{1}, q_{2}, \lambda\right)$ of forms on $\mathbb{C}^{3}$ ( $q_{1}$ and $q_{2}$ quadratic, $\lambda$ linear), whose vanishing is a necessary and sufficient condition for the traces of the conics of equations $q_{1}(x)=0$ and $q_{2}(x)=0$ on the line of equation $\lambda(x)=0$ to be a harmonic system of points.

Set

$$
\Phi_{14}=\sum_{i, j} \frac{\partial \Phi_{5}}{d y_{i} d y_{j}} \Psi\left(f_{i}, f_{j}, \lambda\right)
$$

\footnotetext{
${ }^{10}$ The equation obtained this way is of smaller degree than the one obtained by simply substituting the $y_{i}$ 's with the $f_{i}$ 's in $\Phi_{10}$. Actually, this latter is proportional to the square of $\Phi_{11}$.
} 
where

$$
\lambda(x)=\lambda_{0} x_{0}+\lambda_{1} x_{1}+\lambda_{2} x_{2} .
$$

Then $\Phi_{14}$ is a covariant of degree 8 and type $\operatorname{Pol}_{2}\left(\left(\mathbb{C}^{3}\right)^{*}\right)$. One checks that $\Phi_{14}(f)=0$ is an equation for the conic of $\left(\mathbb{C P}^{2}\right)^{*}$ dual to the conic of equation $\Phi_{13}(f)=0$ of $\mathbb{C P}^{2}$. Note that the equation we find this way has lower degree than the one obtained by computing the comatrix of the matrix of $\Phi_{13}(f)$ (that would have degree 32).

Quadrilateral, preimage of the four torsal conics. The union of the four torsal conics is also the intersection between the Associated Quadric (defined by $\Phi_{5}(f)=0$ ) and the Steiner surface. Thus, its preimage is also the preimage of the quadric.

Substitute $y_{i}$ with $f_{i}$ in $\Phi_{5}$, this gives a new covariant $\Phi_{15}$ of degree 8 and type $\operatorname{Pol}_{4}\left(\mathbb{C}^{3}\right)$ :

$$
\Phi_{15}(f)(x)=\Phi_{5}(f)(f(x)) .
$$

The zero locus of $\Phi_{15}(f)$ in $\mathbb{C P}^{2}$ is the quadrilateral, preimage of the union of the torsal conics.

\section{APPLICATION: EqUations AND INEQUALITIES DEFINING THE TYPES OF STEINER SURFACES}

We want to recognize the orbits in $\mathcal{U}$, that is the orbits of parameterizations of quartic surfaces (from those of surfaces of smaller degree), and next to discriminate between these orbits.

We consider the first task. After [Aries et al., 2004] (Proposition 2 and Proposition 5), there are three cases to rule out. The first case is when the parameterization $[f]$ admits a base point (i.e. the $f_{i}$ 's have a common zero in $\mathbb{C P}^{2}$ ). The second case corresponds to the orbit of the parameterization

$$
\left(x_{0}^{2}: x_{1}^{2}: x_{2}^{2}: x_{1} x_{2}\right) .
$$

The Zariski closure of its image is a quadric. The third case is the case when the Zariski closure of the image of the parametrization is a plane. A necessary and sufficient condition for being in the first case is the identical vanishing of $\Phi_{4}(f)$, which translates into a system of polynomial equations of degree 12 in the coefficients of $f$. The second case is isolated by remarking (by mere evaluation on the representative) that $\Phi_{11}$ vanishes identically on the orbit of (33), and not on the six orbits of parameterizations giving true quartics. This gives another system of equations of degree 12 . The third case is detected by the vanishing of the maximal minors of the $4 \times 6$ matrix of the coefficients of the $f_{i}$ 's. This is a system of equations of degree 4 .

Now we evaluate the covariants of our collection on the representatives of the six orbits in $\mathcal{U}$, and find that $\Phi_{14}$ makes possible the discrimination. Let us explain how: $\Phi_{14}(f)$ is a quadratic form on $\mathbb{R}^{3}$. Let $M(f)$ be its matrix. Then the inertia of $\Phi_{14}(f)$ is the following ordered pair: (number of positive eigenvalues of $M$, number of negative eigenvalues of $M(f)$ ). The covariance property of $\Phi_{14}$ can be stated as follows:

$$
\left(\Phi_{14}\left(\rho \circ f \circ \theta^{-1}\right)\right)(\lambda)=\operatorname{det}(\theta)^{-6} \operatorname{det}(\rho)^{2}\left(\Phi_{14}(f)\right)\left(\lambda \circ \theta^{-1}\right)
$$

Because the powers of the determinants involved in the formulas are even, the inertia of $\Phi_{14}(f)$ takes only one value on each orbit of $\mathcal{F}$ under $G$. As a consequence, it defines a function on $\mathcal{U}$. Table 3 shows its values.

It is already an interesting result that the inertia of one quadratic form attached to $f$ is enough to discriminate between the six orbits in $\mathcal{U}$. 


\begin{tabular}{|c|c|c|}
\hline Orbit of $[f]$ & inertia of $\Phi_{14}(f)$ & equations and inequalities \\
\hline Ii & $(0,3)$ & $A_{3}>0 \wedge A_{2}>0 \wedge A_{1}>0$ \\
Iii & $(2,1)$ & $A_{3}>0 \wedge\left(A_{2} \leq 0 \vee A_{1} \leq 0\right)$ \\
Iiii & $(1,2)$ & $A_{3}<0$ \\
IIi & $(1,1)$ & $A_{3}=0 \wedge A_{2}<0$ \\
IIii & $(0,2)$ & $A_{3}=0 \wedge A_{2}>0$ \\
III & $(0,1)$ & $A_{3}=A_{2}=0$ \\
\hline
\end{tabular}

TABLE 3. Discrimination between the orbits.

Now, we want to go further and define the orbits by equations and inequalities. For this we introduce the characteristic polynomial of $M(f)$ :

$$
\operatorname{det}(t \cdot I-M(f))=t^{3}+A_{1}(f) t^{2}+A_{2}(f) t+A_{3}(f) .
$$

Any condition on the inertia can be translated into equations and inequalities involving the coefficients of $A_{i}(f)$. The formulas obtained are presented in the last column of Table 3 . They are obtained trivially, except those for discriminating between inertias $(2,1)$ and $(0,3)$, that makes use of Descartes' law of signs [Basu et al., 2003].

Note that $A_{3}(f)$ is a non-trivial invariant of degree 24 . Thus it should be proportional to $\Delta$. One finds (by evaluation on the representative of Orbit Ii) that the coefficient of proportionality is positive. Thus in the sign conditions above, we are allowed to substitute $A_{3}$ with $\Delta$.

\section{CONCLUSION}

In this paper, we have produced a collection of covariants for quadratic parameterizations of surfaces. We were guided by the geometry of the Steiner surface. In future work, we wish to tackle the problem in a more systematic way: exploiting methods from Invariant Theory, we will try to produce systems of generators for the covariants; or at least to describe all the covariants of low degree.

Acknowledgments. The authors want to thank the readers and anonymous referees, of this paper and of a previous version, for their constructive remarks.

Emmanuel Briand was supported by the European Research Training Network RAAG (Real Algebraic and Analytic Geometry), contract N ${ }^{\circ}$ HPRN-CT-2001-00271. He wishes to thank the Universidad de Cantabria for its hospitality in 2005.

\section{REFERENCES}

[Apery, 1987] Apery, F. (1987). Models of the Real Projective Plane. Vieweg.

[Aries et al., 2004] Aries, F., Mourrain, B., and Técourt, J.-P. (2004). Quadratically parameterized surfaces: Algorithms and applications. In Geometric Modeling and Computing: Seattle 2003, pages 21-40. Nashboro Press. [Aries and Senoussi, 1997] Aries, F. and Senoussi, R. (1997). Approximation de surfaces paramétriques par des carreaux rationnels du second degré en lancer de rayons. Revue Internationale de CFAO et d'Informatique Graphique, 12:627-645.

[Aries and Senoussi, 2001] Aries, F. and Senoussi, R. (2001). An implicitization algorithm for rational surfaces with no base points. Journal of Symbolic Computation, 31:357-365.

[Basu et al., 2003] Basu, S., Pollack, R., and Roy, M.-F. (2003). Algorithms in real algebraic geometry, volume 10 of Algorithms and Computation in Mathematics. Springer-Verlag, Berlin.

[Brill, 1872] Brill, A. v. (1872). Note über die Gleichung der auf einer Ebene abbildbaren Flächen. Math. Ann., 5:401-403.

[Coffman et al., 1996] Coffman, A., Schwartz, A. J., and Stanton, C. (1996). The algebra and geometry of Steiner and other quadratically parametrizable surfaces. Computer Aided Geometric Design, 13:257-286. 
[Degen, 1996] Degen, W. (1996). The types of triangular Bézier surfaces. In Mullineux, G., editor, The Mathematics of Surfaces IV, volume 38 of The Institute of Mathematics and its Applications Conference, pages 153-171. Clarendon, Oxford.

[España et al., 1999] España, M.-L., Baret, F., Aries, F., Chelle, M., Andrieu, B., and Prévot, L. (1999). Modeling maize canopy 3D architecture: Application to reflectance simulation. Ecological Modeling, 122:25-43.

[Jouanolou, 1996] Jouanolou, J.-P. (1996). Résultants anisotropes : Compléments et applications. The Electronic Journal of Combinatorics, 3(2):1-92.

[Kraft and Procesi, 1996] Kraft, H. and Procesi, C. (1996). Classical invariant theory, a primer. Lecture Notes. Preliminary version.

[Popov and Vinberg, 1994] Popov, V. and Vinberg, E. (1994). Algebraic Geometry IV, volume 55 of Encyclopaedia of Mathematical Science, chapter Invariant Theory. Springer-Verlag.

[Salmon, 1884] Salmon, G. (1884). Traité de Géométrie Analytique à Deux Dimensions, (sections coniques). Gautier-Villars, Paris.

[Salmon, 1915] Salmon, G. (1915). A Treatise on the Analytic Geometry of Three Dimensions. Longman, Greens and co., fifth edition. Revised by Reginald A. P. Rogers.

[Sederberg and Chen, 1995] Sederberg, T. W. and Chen, F. (1995). Implicitization using curves and surfaces. Computer Graphics Siggraph 1995, 18:301-308.

Franck Aries, Claude Bruchou: INRA Biométrie, Avignon (France)

E-mail address: Franck. Aries@avignon.inra.fr

E-mail address: Claude.Bruchou@avignon.inra.fr

Emmanuel BRiand: Universidad de CANTABRia, SANTANDER (SPAin)

E-mail address: emmanuel.briandegmail.com 